Canad. J. Math. Vol. 54 (6), 2002 pp. 1187-1201

\title{
On the Injectivity of $C^{1}$ Maps of the Real Plane
}

\author{
Milton Cobo, Carlos Gutierrez and Jaume Llibre
}

Abstract. Let $X: \mathbb{R}^{2} \rightarrow \mathbb{R}^{2}$ be a $C^{1}$ map. Denote by $\operatorname{Spec}(X)$ the set of (complex) eigenvalues of $\mathrm{DX}_{p}$
when $p$ varies in $\mathbb{R}^{2}$. If there exists $\epsilon>0$ such that $\operatorname{Spec}(X) \cap(-\epsilon, \epsilon)=\varnothing$, then $X$ is injective. Some
applications of this result to the real Keller Jacobian conjecture are discussed.

\section{Introduction}

Let $X: \mathbb{R}^{2} \rightarrow \mathbb{R}^{2}$ be a map of class $C^{1}$. We shall denote by $\operatorname{Spec}(X)$ the set of (complex) eigenvalues of the derivative $\mathrm{DX}_{p}$ when $p$ varies in $\mathbb{R}^{2}$. We will refer to $X: \mathbb{R}^{2} \rightarrow \mathbb{R}^{2}$ as a Keller map if $X$ is a polynomial map and the Jacobian determinant of $X$ is identically equal to one in $\mathbb{R}^{2}$. It is important to observe that if $X: \mathbb{R}^{2} \rightarrow \mathbb{R}^{2}$ is a Keller map, then $\operatorname{Spec}(X) \subset \mathbb{S}^{1} \cup(\mathbb{R} \backslash\{0\})$. The bidimensional Real Keller Conjecture claims that if $X: \mathbb{R}^{2} \rightarrow \mathbb{R}^{2}$ is a Keller map, then $X$ is injective. For more details about Keller maps and the Jacobian conjecture see the recent book of van den Essen [5].

Our main result is the following:

Theorem $A$ Let $X: \mathbb{R}^{2} \rightarrow \mathbb{R}^{2}$ be a $C^{1}$ map. Suppose that, for some $\epsilon>0, \operatorname{Spec}(X) \cap$ $(-\epsilon, \epsilon)=\varnothing$. Then $X$ is injective.

Relevant to this theorem, we may say:

(1) It is optimal because if the assumptions are relaxed to $0 \notin \operatorname{Spec}(X)$, the conclusion - even for a polynomial map $X$-is not true anymore, as shown by Pinchuck's counterexample [15] (See also [5], page 241).

(2) It confirms in a stronger way, the following Chamberland's conjecture [3] in dimension 2: Let $Y: \mathbb{R}^{n} \rightarrow \mathbb{R}^{n}$ be a $C^{1}$ map. Suppose that there exists an $\epsilon>0$ such that, for all $\lambda \in \operatorname{Spec}(Y),|\lambda|>\epsilon$. Then $Y$ is injective.

(3) It does not imply the bidimensional real Keller Conjecture because, given $n$ an even natural, the polynomial Keller map

$$
X(x, y)=\left(-y, x+y^{n}\right)
$$

satisfies $\operatorname{Spec}(X)=\mathbb{S}^{1} \cup(\mathbb{R} \backslash\{0\})$ (that is, $\operatorname{Spec}(X)$ is the biggest possible for Keller maps). This example will be studied in Section 8 .

(4) Campbell [2] classified the two-dimensional $C^{1}$ maps whose eigenvalues are both 1 . All such maps have an explicit inverse. The class of functions considered in Theorem A is much broader, but no explicit inverse is given.

Received by the editors June 27, 2001; revised May 7, 2002.

AMS subject classification: Primary: 34D05; secondary: 54H20, 58F10, 58F21.

(c) Canadian Mathematical Society 2002. 
Theorem A is proved in Section 3. A key point in its proof is notion of half-Reeb components which will be introduced in Section 2 .

The next result is for $C^{1}$-maps having at least one component polynomial of the form $p(x) q(y)$.

Theorem B Let $g: \mathbb{R}^{2} \rightarrow \mathbb{R}$ be a $C^{1}$-map, let $p, q: \mathbb{R} \rightarrow \mathbb{R}$ be polynomial maps and let

$$
X(x, y)=(f(x, y), g(x, y))=(p(x) q(y), g(x, y))
$$

Then if $0 \notin \operatorname{Spec}(X), X$ is injective.

Theorem B is proved in Section 4.

For polynomial maps we have the following results.

Theorem $C$ Let $X: \mathbb{R}^{2} \rightarrow \mathbb{R}^{2}$ be a polynomial map. Suppose that, for some $\epsilon>0$, either $\operatorname{Spec}(X) \cap(-\epsilon, 0]=\varnothing$ or $\operatorname{Spec}(X) \cap[0, \epsilon)=\varnothing$. Then $X$ is injective.

This is a sharper version of Theorem A in the case of polynomial maps.

Theorem $D$ Let $X=(f, g): \mathbb{R}^{2} \rightarrow \mathbb{R}^{2}$ be a polynomial map such that $\operatorname{Spec}(X) \cap$ $\{0\}=\varnothing$ and denote

$$
\Gamma=\left\{(x, y) \in \mathbb{R}^{2}: \operatorname{Trace}(\mathrm{DX})(x, y)=0\right\}
$$

Then the following statements hold.

(a) If $\left.f\right|_{\Gamma}$ or $\left.g\right|_{\Gamma}$ is a proper map, then $X$ is injective.

(b) $X$ is injective if and only if $\left.\left(f^{2}+g^{2}\right)\right|_{\Gamma}$ is a proper map.

Theorems C and D are proved in Section 7. Their proof uses the notion of pair of aligned half-Reeb components which will be introduced in Section 5.

In Section 8 we will give some examples and applications of the results above.

\section{Half-Reeb Components and Injectivity}

Let $f: \mathbb{R}^{2} \rightarrow \mathbb{R}$ be a $C^{1}$ submersion. For $q \in \mathbb{R}^{2}$ we denote by $X_{f}(q)=\left(-f_{y}(q)\right.$, $\left.f_{x}(q)\right)$ the planar Hamiltonian vector field with Hamiltonian $f$. As usual $\nabla f(p)=$ $\left(f_{x}(p), f_{y}(p)\right)$ denotes the gradient of $f$. Let $g(x, y)=x y$ and consider the set

$$
B=\{(x, y) \in[0,2] \times[0,2]: x+y \leq 2\} \backslash\{(0,0)\}
$$

Definition 1 We will say that $\mathcal{A} \subset \mathbb{R}^{2}$ is a half-Reeb component for $X_{f}$ (or simply a $\mathrm{hRc}$ for $X_{f}$ ) if there is a homeomorphism $h: B \rightarrow \mathcal{A}$ which is a topological equivalence between $\left.X_{f}\right|_{\mathcal{A}}$ and $\left.X_{g}\right|_{B}$ and such that

(1) The segment $\{(x, y) \in B: x+y=2\}$ is sent by $h$ onto a transversal section for the flow of $X_{f}$ in the complement of $h(1,1)$; this section is called the compact edge of $\mathcal{A}$. 


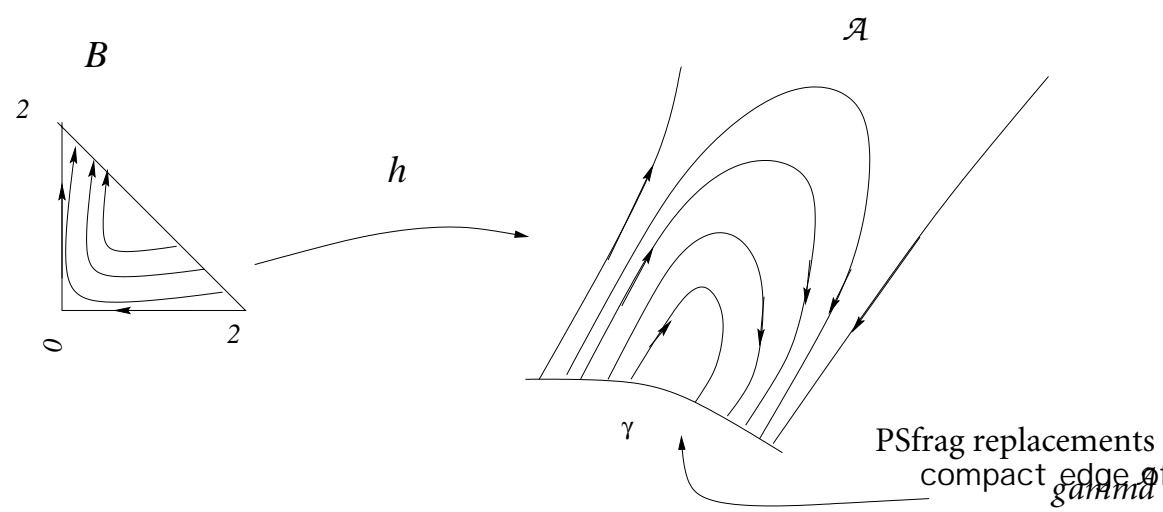

Figure 1: A half-Reeb component.

(2) Both segments $\{(x, y) \in B: x=0\}$ and $\{(x, y) \in B: y=0\}$ are sent by $h$ onto full half-trajectories of $X_{f}$. These two semi-trajectories of $X_{f}$ are called the non-compact edges of $\mathcal{A}$.

The connection between half-Reeb components and injectivity is given by the following result.

Proposition 1 Suppose that $X=(f, g): \mathbb{R}^{2} \rightarrow \mathbb{R}^{2}$ is a $C^{1}$ map such that $0 \notin$ $\operatorname{Spec}(X)$. If $X$ is not injective, then both $X_{f}$ and $X_{g}$ have $\mathrm{hRc}$ 's.

Proof Suppose by contradiction that $X_{f}$ has no half-Reeb components. By assumption, the Hamiltonian vector field $X_{f}$, induced by $f$, has no singularities. Hence, by Kaplan's classification of planar foliations [11], we obtain that $X_{f}$ is topologically equivalent to the horizontal foliation of $\mathbb{R}^{2}$. This and the fact that $f$ is a submersion imply that each nonempty level curve of $f$ must have exactly one connected component. As $g$ restricted to each level curve of $f$ is strictly monotone, we arrive at the contradiction that $X$ is injective. This finishes the proof of the proposition.

For each $\theta \in \mathbb{R}$ let $R_{\theta}$ denote the linear rotation

$$
\left(\begin{array}{rr}
\cos \theta & -\sin \theta \\
\sin \theta & \cos \theta
\end{array}\right)
$$

We will use in the sequel the following proposition.

Proposition 2 Let $X=(f, g): \mathbb{R}^{2} \rightarrow \mathbb{R}^{2}$ be a non-injective $C^{1}$-map such that $0 \notin$ $\operatorname{Spec}(X)$. Let $\mathcal{A}$ be $a \mathrm{hRc}$ of $X_{f}$ and let $\left(f_{\theta}, g_{\theta}\right)=R_{\theta} \circ X \circ R_{-\theta}, \theta \in \mathbb{R}$. Then there is an $\epsilon>0$ such that for all $\theta \in(-\epsilon, 0) \cup(0, \epsilon), X_{f_{\theta}}$ has a hRc whose projection on the $x$-axis is an interval of infinite length.

The proof of this proposition is contained in [10, Lemma 2.5]. 


\section{Proof of Theorem A}

Suppose by contradiction that $X=(f, g)$ is not injective. Hereafter we will use the fact that non-injectivity and the assumptions of Theorem A are open in the Whitney $C^{1}$ topology; in particular we shall assume, from now on, that $X$ is smooth.

By Proposition 1, $X_{f}$ has a half-Reeb component $\mathcal{A}$. Let $\Pi: \mathbb{R}^{2} \rightarrow \mathbb{R}$ be the projection on the first coordinate. By composing with a rotation if necessary, in the way stated in Proposition 2, we may assume that $\Pi(\mathcal{A})$ is an interval of infinite length. To simplify matters, let us suppose that $[b, \infty) \subset \Pi(\mathcal{A})$.

By Thom's Transversality Theorem for jets [7], we can assume the following:

(a1) the set

$$
T=\left\{(x, y) \in \mathbb{R}^{2}: f_{y}(x, y)=0\right\}
$$

is made up of regular curves;

(a2) There is a discrete subset $\Delta$ of $T$ such that if $p \in T \backslash \Delta$ (resp. $p \in \Delta$ ), $X_{f}$ has quadratic contact (resp. cubic contact) with the vertical foliation of $\mathbb{R}^{2}$.

Then, if $a>b$ is large enough,

(b) for any $x \geq a$, the vertical line $\Pi^{-1}(x)$ intersects exactly one trajectory $\alpha_{x} \subset \mathcal{A}$ of $\left.X_{f}\right|_{\mathcal{A}}$ such that $\Pi\left(\alpha_{x}\right) \cap(x, \infty)=\varnothing$; in other words, $x$ is the maximum for the restriction $\left.\Pi\right|_{\alpha_{x}}$.

It follows that

(c) if $x \geq a$ and $p \in \alpha_{x} \cap \Pi^{-1}(x)$ then $p \in T \cap \mathcal{A} \backslash \Delta$.

Let $T_{x}$ be the set of $p \in \mathcal{A}$ such that $p \in \alpha_{x} \cap \Pi^{-1}(x), x \geq a$. Notice that, for every $x \geq a, \alpha_{x} \cap \Pi^{-1}(x)$ is a finite set; nevertheless, by (b), (c) and by using Thom's Transversality Theorem for jets, we may get the following stronger statement:

(d) There is a sequence $F=\left\{a_{1}, a_{2}, \ldots, a_{i}, \ldots\right\}$ in $[a, \infty)$, which may be either empty or finite or else countable, such that if $x \in F$ (resp. $x \in[a, \infty) \backslash F$ ), then $\Pi^{-1}(x) \cap T_{x}$ is a two-point-set (resp. a one-point-set).

If $x \in[a, \infty) \backslash F$, define $\eta(x)=\left(x, \eta_{2}(x)\right)=\Pi^{-1}(x) \cap T_{m}$. Observe that $\eta:[a, \infty) \backslash F \rightarrow T_{m}$ is a smooth embedding. As $\left.f\right|_{\mathcal{A}}$ is bounded,

(e) $F \circ \eta$ extends continuously to a strictly increasing bounded map defined in $[a, \infty)$ such that, for all $x \in[a, \infty) \backslash F, f_{x}(\eta(x))$ has constant sign.

Therefore, there exists a real constant $K$ such that

$$
\begin{aligned}
K & =\int_{a_{1}}^{\infty} \frac{d}{d x} f(\eta(x)) d x=\sum_{i=1}^{\infty} \int_{a_{i}}^{a_{i+1}} \frac{d}{d x} f(\eta(x)) d x \\
& =\sum_{i=1}^{\infty} \int_{a_{i}}^{a_{i+1}} f_{x}(\eta(x))
\end{aligned}
$$

This and (e) imply that, for some sequence $x_{n} \rightarrow \infty, \lim _{n \rightarrow \infty} f_{x}\left(\eta\left(x_{n}\right)\right)=0$. This is a contradiction with the assumption $\operatorname{Spec}(X) \cap(-\epsilon, \epsilon)=\varnothing$. In short we have proved Theorem A.

\section{Proof of Theorem B}

By the assumptions we have that 
(a) $f_{x}(x, y)=p^{\prime}(x) q(y)$ and $f_{y}(x, y)=p(x) q^{\prime}(y)$

As $0 \notin \operatorname{Spec}(X)$, we obtain that

(b) for all $y \in \mathbb{R}$, either $q(y) \neq 0$ or $q^{\prime}(y) \neq 0$.

Now we assume, by contradiction, that $X$ is not injective; then, by Proposition 1 , there is a half-Reeb component $\mathcal{A}$ for $X_{f}$. As $0 \notin \operatorname{Spec}(X), f$ is a submersion and so the non-compact edges of $\mathcal{A}$ must accumulate at infinity. This implies that the projection of $\mathcal{A}$ on at least one of the coordinate axis has infinite length; let us consider only the case in which this happens for the $x$-axis. Let $T=\left\{w \in \mathbb{R}^{2}: f_{y}(w)=0\right\}$. Similarly to the proof of Theorem A, we obtain that the projection of $T \cap \mathcal{A}$ on the $x$-axis is an interval of infinite length, say $[a, \infty)$. Let $G=\left\{y_{1}, y_{2}, \ldots, y_{m}\right\}$ and $F=\left\{x_{1}, x_{2}, \ldots, x_{n}\right\}$ be all the real roots of the polynomials $q^{\prime}(y)$ and $p(x)$ respectively. Under these conditions, using (a) and (b), we obtain,

(c) if $(x, y) \in T \cap \mathcal{A}$ and $x \notin F$, then $y \in G$ and $q(y) \neq 0$.

Since $f$ restricted to $\mathcal{A}$ is bounded, there is a constant $M>0$ such that, for all $(x, y) \in \mathcal{A},|f(x, y)=p(x) q(y)| \leq M$. Therefore, by (c), if $(x, y) \in T \cap \mathcal{A}$, and $x \notin F$, we have that

$$
|p(x)| \leq \frac{M}{\min _{y_{i} \in G}\left|q\left(y_{i}\right)\right|},
$$

which implies that $p(x)$ is identically constant. Hence, for all $(x, y) \in \mathbb{R} \times G$, $f_{x}(x, y)=f_{y}(x, y)=0$, and so $0 \in \operatorname{Spec}(X)$. This contradiction proves that $X$ is injective.

\section{Aligned and Adjacent Half-Reeb Components}

Definition 2 Let $\gamma=\left(\gamma_{1}, \gamma_{2}\right):[0,3] \rightarrow \mathbb{R}^{2}$ be a compact edge of a half-Reeb component $\mathcal{A}$ for $X_{f}$ and let $\gamma\left(t_{0}\right)$ be the unique point where the curve $\gamma$ is tangent to the flow $X_{f}$. Consider the vector $\dot{\gamma}\left(t_{0}\right)^{\perp}=\left(-\dot{\gamma}_{2}\left(t_{0}\right) \dot{\gamma}_{1}\left(t_{0}\right)\right)$ and the straight line $r(s)=s \dot{\gamma}\left(t_{0}\right)^{\perp}+\gamma\left(t_{0}\right), s \in \mathbb{R}$, passing through $\gamma\left(t_{0}\right)$ with direction $\dot{\gamma}\left(t_{0}\right)^{\perp}$. We will say that $\mathcal{A}$ is on the left (resp. on the right) of $\gamma$, if there is an interval $[0, \delta$ ) (resp. $(-\delta, 0])$ such that $r([0, \delta)) \subset \mathcal{A}($ resp. $r((-\delta, 0]) \subset \mathcal{A}))$.

In order to prove Theorems $\mathrm{C}$ and $\mathrm{D}$, we need to introduce some definitions and state some results. First at all we introduce the notion of aligned half-Reeb components.

Definition 3 Let $f: \mathbb{R}^{2} \rightarrow \mathbb{R}$ be a $C^{1}$ submersion and let $\mathcal{A}$ and $\mathcal{B}$ be disjoint halfReeb components for $X_{f}$. We say that $\mathcal{A}$ and $\mathcal{B}$ are aligned and denote $\{\mathcal{A}, \mathcal{B}\}$, if there is a smooth embedded curve $\gamma:[1,2] \rightarrow \mathbb{R}^{2}$ such that the following properties are satisfied:

(a1) For some $1<s_{0} \leq r_{0}<2,\left.\gamma\right|_{\left[s_{0}, r_{0}\right]}$ is transversal to $X_{f}$;

(a2) $\gamma\left(\left[1, s_{0}\right]\right)$ and $\gamma\left(\left[r_{0}, 2\right]\right)$ are the compact edges for $\mathcal{A}$ and $\mathcal{B}$, respectively, and $\gamma\left(\left(s_{0}, r_{0}\right)\right)$ is disjoint of $\mathcal{A}$ and $\mathcal{B}$;

(a3) $\mathcal{A}$ and $\mathcal{B}$ are both either on the left or on the right of the curve $\gamma$. 
The curve $\gamma$ will be said to be an aligning path for the pair $\{\mathcal{A}, \mathcal{B}\}$ with connecting interval $\left[s_{0}, r_{0}\right]$. See Figure 2. Let $\alpha_{p}^{+}$(resp. $\alpha_{p}^{-}$) denote the positive (resp. negative) half-trajectory of $X_{f}$ starting at $p \in \mathbb{R}^{2}$. If $\alpha_{\gamma\left(s_{0}\right)}^{+}$and $\alpha_{\gamma\left(r_{0}\right)}^{+}\left(\right.$resp. $\alpha_{\gamma\left(s_{0}\right)}^{-}$and $\left.\alpha_{\gamma\left(r_{0}\right)}^{-}\right)$ are non-compact edges of $\mathcal{A}$ and $\mathcal{B}$, respectively, then $\gamma$, as right above, will be said to be a positive (resp. negative) aligning path for the pair $\mathcal{A}$ and $\mathcal{B}$.

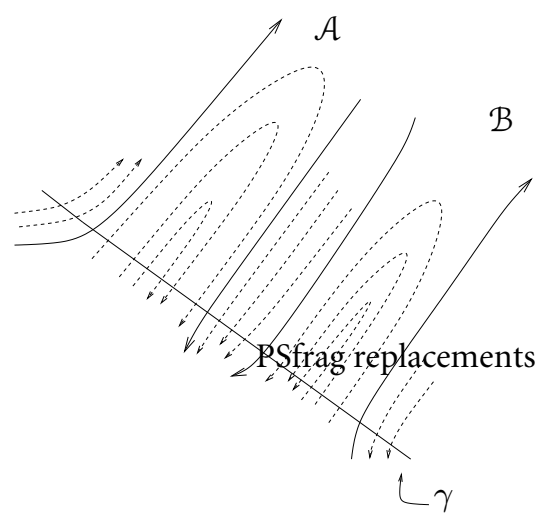

Figure 2: A pair of aligned half Reeb components

We shall need the following result of Gutierrez (see [10, Theorem D]):

Proposition 3 Suppose that $X: \mathbb{R}^{2} \rightarrow \mathbb{R}^{2}$ is a $C^{1}$ map such that $0 \notin \operatorname{Spec}(X)$ and, for all $\theta \in \mathbb{R}, X_{f_{\theta}}$ has no pair of aligned hRc's, where $\left(f_{\theta}, g_{\theta}\right)=R_{\theta} \circ X \circ R_{-\theta}$. Then, $X$ is injective.

To introduce the notion of adjacent half-Reeb components we will consider the following compactification of the plane.

Let $\mathbb{S}^{2}=\left\{(x, y, z) \in \mathbb{R}^{3}: x^{2}+y^{2}+(z-1)^{2}=1\right\}, \mathbb{S}_{-}^{2}=\left\{(x, y, z) \in \mathbb{S}^{2}: 0 \leq z<\right.$ $1\}$, and $\mathbb{S}^{1}=\left\{(x, y, 1) \in \mathbb{S}^{2}\right\}$. Let $\varphi: \mathbb{S}^{2} \backslash \mathbb{S}^{1} \rightarrow \mathbb{R}^{2}$ be the 2-to-1 map given by

$$
\varphi(x, y, z)=\left(\frac{x}{1-z}, \frac{y}{1-z}\right) .
$$

We shall denote by $\mathrm{Cl}\left(\mathbb{R}^{2}\right)$ the compact disc made up of the union of $\mathbb{R}^{2}$ and $\mathbb{S}^{1}$ by identifying $(x, y, z) \in \mathbb{S}_{-}^{2}$ with $\varphi(x, y, z)$ and by borrowing from $\overline{\mathbb{S}_{-}^{2}}=\mathbb{S}_{-}^{2} \cup \mathbb{S}^{1}$ its topology.

If $f: \mathbb{R}^{2} \rightarrow \mathbb{R}$ is a submersion then given a half trajectory $\alpha$ of $X_{f}$, it follows from the arguments of the Poincaré-Bendixson Theorem and from the fact that $X_{f}$ has no singularities, that the limit set

$$
\mathcal{L}(\alpha):=\bar{\alpha} \backslash \alpha
$$

of $\alpha$-as a subset of $\mathrm{Cl}\left(\mathbb{R}^{2}\right)$ - , is either $\mathbb{S}^{1}$ or a nonempty closed sub-interval of it. Also if $\mathcal{A}$ is a half-Reeb component of $X_{f}$, the difference $\mathcal{L}(\mathcal{A}):=\overline{\mathcal{A}} \backslash \mathcal{A}\left(\right.$ in $\mathrm{Cl}\left(\mathbb{R}^{2}\right)$ ) is 
a compact connected subset of $\mathrm{Cl}\left(\mathbb{R}^{2}\right)$ which may or may not be properly contained in $\mathbb{S}^{1}$.

Given $p \in \mathbb{R}^{2}$ we will denote by $\alpha_{p}$ the trajectory of $X_{f}$ passing through $p$. Also, $\alpha_{p}^{+}$and $\alpha_{p}^{-}$will denote the positive and negative half-trajectories of $X_{f}$, respectively, starting at $p$. If $\gamma:[a, b] \rightarrow \mathbb{R}^{2}$ is an embedded smooth curve transversal to $X_{f}$ and and $a \leq t_{1} \leq t_{2} \leq b$, we will denote

$$
\mathcal{R}^{+}\left(\gamma\left(\left[t_{1}, t_{2}\right]\right)\right)=\bigcup_{t_{1} \leq s \leq t_{2}} \alpha_{\gamma(s)}^{+}, \quad \mathcal{R}^{-}\left(\gamma\left(\left[t_{1}, t_{2}\right]\right)\right)=\bigcup_{t_{1} \leq s \leq t_{2}} \alpha_{\gamma(s)}^{-} .
$$

Definition 4 Let $\mathcal{A}$ and $\mathcal{B}$ be a pair of aligned hRc's for $X_{f}$ and let $\gamma:[1,2] \rightarrow \mathbb{R}^{2}$ be an aligning path for the pair $\{\mathcal{A}, \mathcal{B}\}$ with connecting interval $\left[s_{0}, r_{0}\right]$. We say that $\mathcal{A}$ and $\mathcal{B}$ are adjacent half-Reeb components for $X_{f}$ if the set

$$
\mathcal{L}\left(\mathcal{R}^{+}\left(\gamma\left(\left[s_{0}, r_{0}\right]\right)\right)\right)=\overline{\bigcup_{s_{0} \leq s \leq r_{0}} \alpha_{\gamma(s)}^{+}} \backslash \bigcup_{s_{0} \leq s \leq r_{0}} \alpha_{\gamma(s)}^{+}
$$

is contained in the unit circle $\mathbb{S}^{1}$ at infinity (resp. $\left.\mathcal{L}\left(\mathcal{R}^{-}\left(\gamma\left(\left[s_{0}, r_{0}\right]\right)\right)\right) \subset \mathbb{S}^{1}\right)$. Set $\Omega=\mathcal{A} \cup \mathcal{R}^{+}\left(\gamma\left[s_{0}, r_{0}\right]\right) \cup \mathcal{B}$ (resp. $\left.\Omega=\mathcal{A} \cup \mathcal{R}^{-}\left(\gamma\left[s_{0}, r_{0}\right]\right) \cup \mathcal{B}\right)$. Notice that $\mathcal{A}$ and $\mathcal{B}$ are adjacent if and only if the subset $\Omega \cup \mathcal{L}(\Omega)$ of $\mathrm{Cl}\left(\mathbb{R}^{2}\right)$ is homeomorphic to a bidimensional compact disc.

\section{Polynomial Maps}

Hereafter we will consider only polynomial maps of the plane. If $f: \mathbb{R}^{2} \rightarrow \mathbb{R}$ is a polynomial submersion, then it may be seen that

(a) $\mathcal{L}\left(\mathcal{R}^{+}\left(\gamma\left[s_{0}, r_{0}\right]\right)\right)$ is just one point of $\mathbb{S}^{1}$; in fact, otherwise, $\mathcal{L}\left(\mathcal{R}^{+}\left(\gamma\left[s_{0}, r_{0}\right]\right)\right)$ would contain an open subinterval $I \subset \mathbb{S}^{1}$ which in turn would imply the contradiction that $f$ is bounded along every ray approaching $I$ (see Figure 3 ).

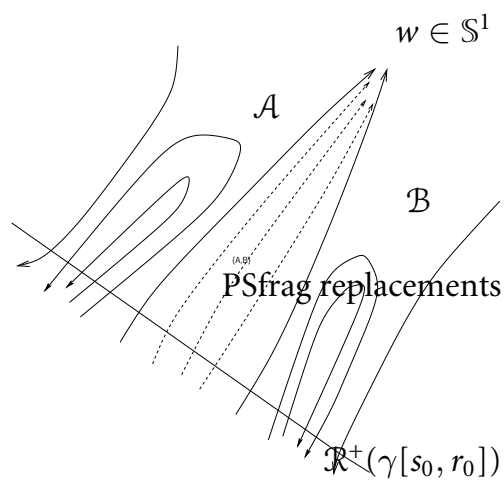

Figure 3: A pair of adjacent half Reeb components 
(b) There exists a finite set $S_{f}=\left\{z_{1}, \ldots, z_{k}\right\}$ of points in the unit circle $\mathbb{S}^{1}$ at infinity such that if $\alpha$ is a half-trajectory of $X_{f}$ then $\mathcal{L}(\alpha)=z_{i}$ for some $i \in\{1, \ldots, k\}$.

(c) If $\mathcal{A}$ is a hRc of $X_{f}$ then $\mathcal{L}(\mathcal{A}) \cap \mathbb{S}^{1} \subset S_{f}$. Each point $z_{i} \in S_{f}$ will be called a limit point for $X_{f}$.

Furthermore, if $\varphi: \mathbb{S}^{2} \backslash \mathbb{S}^{1} \rightarrow \mathbb{R}^{2}$ is the map defined by equation (1), by studying the Poincaré compactification $\tilde{X}_{f}$ —via $\varphi$ —of the polynomial vector field $X_{f}$, we may conclude, using Dumortier's work [4], that

(d) $X_{f}$ has finitely many hRc's.

Within the proof of next result we will use the following notation. If $\gamma \subset \mathrm{Cl}\left(\mathbb{R}^{2}\right)$ is an arc and $p, q \in \gamma$ we denote by $[p, q]_{\gamma}$ (resp. $\left.(p, q)_{\gamma}\right)$ the closed (resp. open) sub-interval of $\gamma$ with endpoints $p$ and $q$.

Proposition 4 Let $X=(f, g): \mathbb{R}^{2} \rightarrow \mathbb{R}^{2}$ be a non-injective polynomial map such that $0 \notin \operatorname{Spec}(X)$. Then there exists $\theta \in \mathbb{R}$ such that $X_{f_{\theta}}$ has a pair $\{\mathcal{A}, \mathcal{B}\}$ of adjacent half-Reeb components such that $e^{ \pm \frac{\pi}{2} i} \notin \mathcal{L}(\mathcal{A}) \cup \mathcal{L}(\mathcal{B})$, where $\left(f_{\theta}, g_{\theta}\right)=R_{\theta} \circ X \circ R_{-}$.

Proof By Proposition 3 there exists $\mu \in \mathbb{R}$ such that $X_{f_{\mu}}$ has a pair of aligned hRc's. Proceed assuming that $f=f_{\mu}$ and consider the case in which the limit set of one of the hRc's of $X_{f}$ contains $e^{ \pm \frac{\pi}{2} i}$.

By [10, Lemma 2.5], we may find $\epsilon>0$ small, such that if $\theta \in(-\epsilon,+\epsilon)$, then $X_{f_{\theta}}$ has a pair $\left\{\mathcal{A}_{\theta}, \mathcal{B}_{\theta}\right\}$ of aligned hRc's. We claim that

(a) if $\epsilon>0$ is small enough, for all $\theta \in(-\epsilon, 0) \cup(0, \epsilon)$ the limit set of both $\mathcal{A}_{\theta}$ and $\mathcal{B}_{\theta}$ is disjoint of $e^{ \pm \frac{\pi}{2} i}$.

In fact, let $f_{n}$ and $g_{k}$ be the highest degree homogeneous part of $f$ and $g$ respectively and let $L_{\theta}$ denote the straight line passing through the origin with slope $\tan (\pi / 2+\theta)$. The assumptions imply that $f_{n}\left(L_{0}\right) \equiv g_{k}\left(L_{0}\right) \equiv 0$. Also, it may be seen that

(b) if $\epsilon>0$ is small and $\theta \in(-\epsilon, 0) \cup(0, \epsilon)$, then both, $f_{n}\left(L_{\theta} \backslash\{0\}\right)$ and $g_{k}\left(L_{\theta} \backslash\{0\}\right)$ are disjoint of $\{0\}$.

To fix ideas, suppose that $n \geq k$. In this way, if $\theta \neq 0$ is small and $\left(f_{\theta}\right)_{n}$ and $\left(g_{\theta}\right)_{n}$ denote the highest degree homogeneous part of $f_{\theta}$ and $g_{\theta}$, respectively, then

$$
\begin{aligned}
& \left(f_{\theta}\right)_{n}=(\cos \theta) f_{n} \circ R_{-\theta}-[k / n](\sin \theta) g_{k} \circ R_{-\theta} \\
& \left(g_{\theta}\right)_{n}=(\sin \theta) f_{n} \circ R_{-\theta}+[k / n](\cos \theta) g_{k} \circ R_{-\theta}
\end{aligned}
$$

where $[k / n]$ denotes the integer part of $k / n$. If we assumed that for some $\theta \in$ $(-\epsilon, 0) \cup(0, \epsilon) \mathcal{A}_{\theta}$ accumulated at infinity at a direction corresponding to $L_{0}$, then we would conclude that $\left(f_{\theta}\right)_{n}\left(L_{0}\right) \equiv 0$. As $g_{\theta}$, restricted to $\mathcal{A}$, is bounded, $\left(g_{\theta}\right)_{n}\left(L_{0}\right) \equiv 0$. Therefore, we would obtain

$$
\left.f_{n}\left(L_{-\theta}\right)=f_{n} \circ R_{-\theta}\left(L_{0}\right)\right)=0=g_{n} \circ R_{-\theta}\left(L_{0}\right)=g_{n}\left(L_{-\theta}\right) .
$$

This contradiction with (b) proves (a).

Let $\theta \in(\mu-\epsilon, \mu+\epsilon)$ be such that $X_{f_{\theta}}$ has a pair $\mathcal{A}_{1}$ and $\mathcal{A}_{2}$ of aligned hRc's with $\mathrm{e}^{ \pm \frac{\pi}{2} i} \notin \mathcal{L}\left(\mathcal{A}_{1}\right) \cup \mathcal{L}\left(\mathcal{A}_{2}\right)$. Let $\gamma:[1,2] \rightarrow \mathbb{R}^{2}$ be a smooth aligning path for $\mathcal{A}_{1}$ and $\mathcal{A}_{2}$, with connecting interval $\left[s_{0} . r_{0}\right]$, which we shall assume to be positive. 
We will prove that if $\mathcal{A}_{1}$ and $\mathcal{A}_{2}$ are not already adjacent, then we can construct a new pair $\left\{\mathcal{B}_{1}, \mathcal{B}_{2}\right\}$ of aligned half-Reeb components for $X_{f_{\theta}}$ such that $\mathcal{B}_{1}$ is either equal to $\mathcal{A}_{1}$ or $\mathcal{A}_{2}$ and the interior of $\mathcal{B}_{2}$ is contained in $\mathcal{R}^{+}\left(\gamma\left[s_{0}, r_{0}\right]\right)$, i.e., $\mathcal{B}_{2}$ is different from $\mathcal{A}_{1}$ and $\mathcal{A}_{2}$. As $f_{\theta}$ is polynomial, there are only a finitely many hRc's for $X_{f_{\theta}}$ and so, proceeding inductively, we will, eventually, arrive to a pair of adjacent hRc's for $X_{f_{\theta}}$. fying

Let $p \in \mathbb{R}^{2} \cap \mathcal{L}\left(\mathcal{R}^{+}\left(\gamma\left[s_{0}, r_{0}\right]\right)\right)$ and let $\eta:[-1,1] \rightarrow \mathbb{R}^{2}$ be a regular curve satis-

$$
\eta(0)=p \quad \text { and } \quad \dot{\eta}(0)=\nabla f(p) .
$$

We may assume that $\left.f\right|_{\eta}$ is strictly monotone and that $\mathcal{R}^{+}\left(\gamma\left[s_{0}, r_{0}\right]\right)$ intersects $\eta$ but $\alpha_{s_{0}}^{+}$and $\alpha_{r_{0}}^{+}$do not intersect $\eta$. Let $s_{0}<t_{1}<t_{2}<t_{0}$ be numbers such that $\alpha_{\gamma\left(t_{1}\right)}^{+}$ and $\alpha_{\gamma\left(t_{2}\right)}^{+}$intersect the curve $\eta$ at points $p_{1}$ and $p_{2}$ respectively. As $f$ restricted to $\left[p_{1}, p_{2}\right]_{\eta}$ and $\left[\gamma\left(t_{1}\right), \gamma\left(t_{2}\right)\right]_{\gamma}$ is strictly monotonous, there is a flow box between the trajectories $\alpha_{t_{1}}^{+}$and $\alpha_{t_{2}}^{+}$and the arcs $\left[p_{1}, p_{2}\right]_{\eta}$ and $\left[\gamma\left(t_{1}\right), \gamma\left(t_{2}\right)\right]_{\gamma}$. In particular, $\left[p_{1}, p_{2}\right]_{\eta} \subset \mathcal{R}^{+}\left(\gamma\left[s_{0}, r_{0}\right]\right)$ and all the semi-trajectories $\alpha_{\gamma(s)}^{+}$with $s \in\left[t_{1}, t_{2}\right]$ intersect (only) once the curve $\eta$ inside the arc $\left[p_{1}, p_{2}\right]_{\eta}$. Therefore $p \notin\left[p_{1}, p_{2}\right]_{\eta}$. Consider only the case in which $p_{1} \in\left[p_{2}, p\right]_{\eta}$ (resp. $\left.p_{2} \in\left[p_{1}, p\right]_{\eta}\right), p_{1}=\eta\left(d_{1}\right)$ and $p_{2}=\eta\left(d_{2}\right)$ with $-1<d_{2}<d_{1}<0$. See Figure 4 .

It is easy to see that we can construct a smooth curve $\eta_{0}$ linking the points $\gamma\left(t_{1}\right)$ and $p_{1}$ (resp. $\gamma\left(t_{2}\right)$ and $p_{2}$ ) and having only one quadratic tangency with the foliation of $X_{f_{\theta}}$. Moreover, $\eta_{0}$ can be chosen in such a way that the curve $\zeta=\left[\gamma(0), \gamma\left(t_{1}\right)\right]_{\gamma} \cup$ $\eta_{0} \cup\left[p_{1}, p\right]_{\eta}$ (resp. $\left.\zeta=\left[\gamma\left(t_{2}\right), \gamma(3)\right]_{\gamma} \cup \eta_{0} \cup\left[p_{2}, p\right]_{\eta}\right)$ is smooth. Observe that $\zeta$ has exactly two (quadratic) tangency points with the foliation of $X_{f_{\theta}}$, one inside the $\operatorname{arc}\left[\gamma(0), \gamma\left(t_{1}\right)\right]_{\gamma}$ (resp. $\left.\left[\gamma\left(t_{2}\right), \gamma(3)\right]_{\gamma}\right)$ corresponding to $\mathcal{A}_{1}$ (resp. $\left.\mathcal{A}_{2}\right)$ and the other inside the curve $\eta_{0}$. Let $t_{0}$ be the supremum of the number $s_{0}<t<t_{1}$ such that $\alpha_{\gamma(t)}^{+}$ intersect the curve $\eta$. By the assumptions $s_{0}<t_{0}<t_{1}$ and $\alpha_{\gamma\left(t_{0}\right)}^{+} \cap \eta=\varnothing$.

Let

$$
d_{0}=\sup \left\{d_{1}<d<0: \eta(d) \in \mathcal{R}^{+}\left(\gamma\left[s_{0}, r_{0}\right]\right)\right\}
$$

Clearly the trajectory $\alpha_{\eta\left(d_{0}\right)}$ does not intersect the arc $\left[\gamma\left(s_{0}\right), \gamma\left(r_{0}\right)\right]_{\gamma}$.

It is easy to see there is a new $\mathrm{hRc} \mathcal{B}_{2}$ of $X_{f_{\theta}}$ whose compact edge is the arc $\left[\gamma\left(t_{0}\right), \eta\left(d_{0}\right)\right]_{\zeta}$ and whose non-compact edges are $\alpha_{\gamma\left(t_{0}\right)}^{+}$and $\alpha_{\eta\left(d_{0}\right)}^{-}$. Clearly the pair $\left\{\mathcal{A}_{1}, \mathcal{B}_{2}\right\}$ (resp. $\left\{\mathcal{A}_{2}, \mathcal{B}_{2}\right\}$ ) is aligned in the sense defined before. This concludes the proof of the proposition.

We shall need the following well known result essentially due to Hadamard (see for more details [5]).

Proposition 5 Let $X=(f, g): \mathbb{R}^{2} \rightarrow \mathbb{R}^{2}$ be a polynomial map such that $\operatorname{Spec}(X) \cap$ $\{0\}=\varnothing$. Then $X$ is a diffeomorphism if and only if $f^{2}+g^{2}: \mathbb{R}^{2} \rightarrow \mathbb{R}$ is a proper map.

We will see in Section 8, that Theorem $D$ give us a better criterium, than that of Proposition 5 to find out whether a polynomial map such that $\operatorname{Spec}(X) \cap\{0\}=\varnothing$ is injective. 


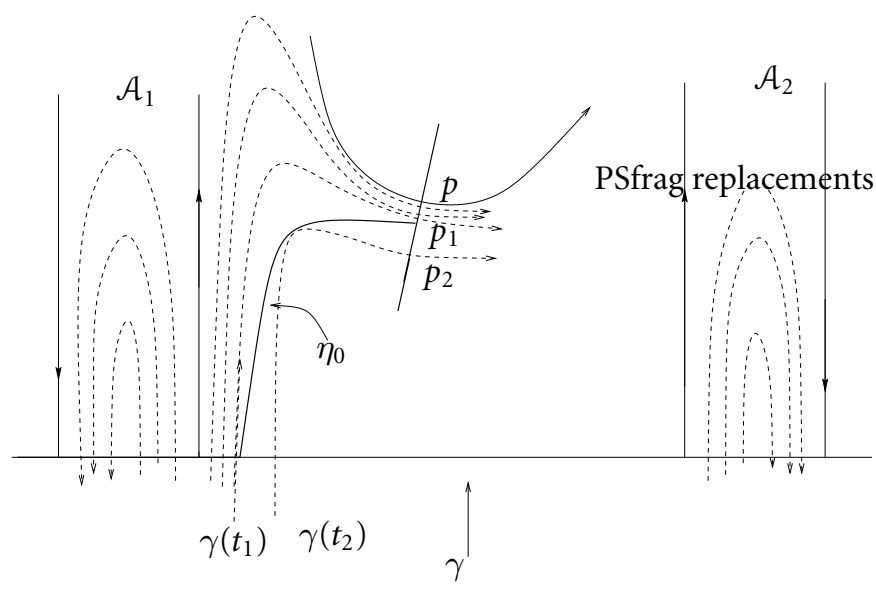

Figure 4: The curve $\eta_{0}$.

\section{Proof of Theorems C and D}

Let us set up the preliminaries for the proof of Theorems $C$ and D. First of all, if $X$ is a non-injective polynomial map then, by Proposition 4 , there exists $\theta \in \mathbb{R}$ such that if $X_{\theta}=\left(f_{\theta}, g_{\theta}\right)=R_{\theta} \circ X \circ R_{-\theta}$, the flow $X_{f_{\theta}}$ has a pair $\left\{\mathcal{A}_{1}, \mathcal{A}_{2}\right\}$ of adjacent hRc's such that $\mathrm{e}^{ \pm \frac{\pi}{2} i} \notin \mathcal{L}\left(\mathcal{A}_{1}\right) \cup \mathcal{L}\left(\mathcal{A}_{2}\right)$. Notice that $\operatorname{Spec}\left(X_{\theta}\right)=\operatorname{Spec}(X)$. As $f_{\theta}$ is polynomial and $\mathcal{A}_{1}$ and $\mathcal{A}_{2}$ are adjacent, $\mathcal{L}\left(\mathcal{A}_{1}\right) \cap \mathcal{L}\left(\mathcal{A}_{2}\right)=\{w\} \subset \mathbb{S}^{1}$, with $w \neq \mathrm{e}^{ \pm \frac{\pi}{2} i}$. Therefore, Proposition 2 implies $\Pi\left(\mathcal{A}_{1}\right) \cap \Pi\left(\mathcal{A}_{2}\right)$ contains an interval of infinite length, where $\Pi: \mathbb{R}^{2} \rightarrow \mathbb{R}$ is the projection on the first coordinate. To fix ideas, let us suppose that $\Pi\left(\mathcal{A}_{1}\right) \cap \Pi\left(\mathcal{A}_{2}\right)$ contains the interval $[b, \infty)$, see figure 5 .

As $f_{\theta}$ is a polynomial map,

(a) the algebraic curve

$$
T_{\theta}=\left\{p \in \mathbb{R}^{2}:\left(f_{\theta}\right)_{y}(p)=0\right\}
$$

is made up of finitely many regular curves and finitely many singular points.

Similarly to the proof of Theorem A, if $a>b$ is large enough, we have that

(b) For any $x \geq a$, the vertical line $\Pi^{-1}(x)$ intersects exactly one trajectory $\alpha_{x}^{i} \subset$ $\mathcal{A}_{i}$ of $\left.X_{f_{\theta}}\right|_{\mathcal{A}_{i}}, i=1,2$, such that $\Pi\left(\alpha_{x}^{i}\right) \cap(x, \infty)=\varnothing, i=1,2$; in other words, $x$ is the maximum for the restriction $\left.\Pi\right|_{\alpha_{x}^{i}}, i=1,2$.

It follows that

(c) If $x \geq a$ and $p_{i} \in \alpha_{x}^{i} \cap \Pi^{-1}(x)$, for $i=1,2$, then there is a "parabolic" tangency between $X_{f_{\theta}}$ and $\Pi^{-1}(x)$ at $p_{i}$. In particular, $p_{i} \in T$, i.e., $\frac{\partial f_{\theta}}{\partial y}\left(p_{i}\right)=0$.

Let $T_{x}^{i}, i=1,2$ be the set of $p \in \mathcal{A}_{i}$ such that $p \in \alpha_{x}^{i} \cap \Pi^{-1}(x), x \geq a$. Notice that, for every $x \geq a, T_{x}^{i}$ is a finite set. By using (a) we may define analytic functions $\eta_{i}:[a, \infty) \rightarrow \mathcal{A}_{i}, i=1,2$ in such a way that $\eta_{i}(x) \in T_{x}^{i}$ and there are no other points of $T_{x}^{i}$ inside the arc of $\Pi^{-1}(x)$ connecting $\eta_{1}(x)$ and $\eta_{2}(x)$. In this way, as the flow $X_{f}$ is continuous (see Figure 5) 
(d) The collinear vectors $\nabla f_{\theta}\left(\eta_{1}(x)\right)$ and $\nabla f_{\theta}\left(\eta_{2}(x)\right)$ have opposed orientations; i.e., $\frac{\partial f_{\theta}}{\partial x}\left(\eta_{1}(x)\right) \cdot \frac{\partial f_{\theta}}{\partial x}\left(\eta_{2}(x)\right)<0$.

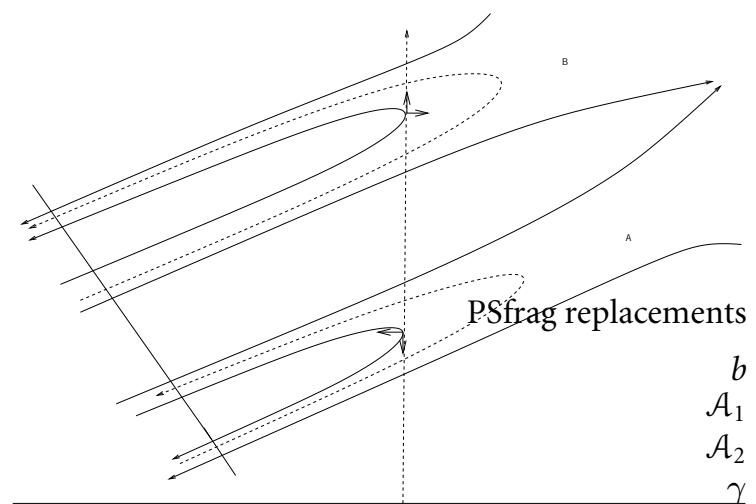

Figure 5: $[b, \infty) \subset \Pi\left(\mathcal{A}_{1}\right) \cap \Pi\left(\mathcal{A}_{2}\right)$.

\subsection{Proof of Theorem C}

If $X$ is a non-injective polynomial map, proceeding as in the proof of Theorem A, we obtain that

$$
\infty>\int_{[a, \infty)} \frac{d}{d s} f_{\theta}\left(\eta_{i}(s)\right) d s=\int_{[a, \infty)}\left(f_{\theta}\right)_{x}\left(\eta_{i}(s)\right) d s, \quad i=1,2,
$$

which implies that $\lim _{n \rightarrow \infty}\left(f_{\theta}\right)_{x}\left(\eta_{1}\left(x_{n}\right)\right)=0$ and $\lim _{n \rightarrow \infty}\left(f_{\theta}\right)_{x}\left(\eta_{2}\left(y_{n}\right)\right)=0$, for some sequences $x_{n}, y_{n} \rightarrow \infty$. This fact together with (d) imply that there are positive and negative values of $\operatorname{Spec}\left(X_{\theta}\right)(=\operatorname{Spec}(X))$ arbitrarily close to zero. Therefore if-for some $\epsilon>0$-either $(-\epsilon, 0] \cap \operatorname{Spec}(X)=\varnothing$ or $\operatorname{Spec}(X) \cap[0, \epsilon)=\varnothing, X$ is injective.

\subsection{Froof of Theorem D}

If $X$ is a non-injective polynomial map such that $\operatorname{Spec}(X) \cap\{0\}=\varnothing$ then the map $X_{\theta}=R_{\theta} \circ X \circ R_{-\theta}$ is also a non-injective polynomial map such that $\operatorname{Spec}(X) \cap$ $\{0\}=\varnothing$ and the condition (d) implies that the signs of $\operatorname{Trace}\left(\operatorname{DX}_{\theta}\left(\eta_{1}(x)\right)\right)$ and $\operatorname{Trace}\left(\operatorname{DX}_{\theta}\left(\eta_{2}(x)\right)\right)$ are opposed. Henceforth, for each $x \geq a$ there is a point $(x, q(x))$ in the arc joining $\eta_{1}(x)$ and $\eta_{2}(x)$ which belongs to the set

$$
\Gamma_{\theta}=\left\{p \in \mathbb{R}^{2}: \operatorname{Trace}\left(\mathrm{DX}_{\theta}\right)(p)=0\right\}
$$


Let $\gamma:[1,2] \rightarrow \mathbb{R}^{2}$ be an aligning path for $\mathcal{A}_{1}$ and $\mathcal{A}_{2}$ as in Definition 3. To fix ideas let us suppose that $X_{f_{\theta}}$ is oriented in such a way that $\alpha_{\gamma\left(s_{0}\right)}^{+}$and $\alpha_{\gamma\left(r_{0}\right)}^{+}$belong to $\mathcal{A}_{1}$ and $\mathcal{A}_{2}$ respectively. In this way, the curve $\Sigma_{\theta}=\{(x, q(x)): x \geq a\} \subset \Gamma_{\theta}$ accumulates at infinity inside the region $\Omega=\mathcal{A}_{1} \cup \mathcal{R}^{+}\left(\gamma\left[s_{0}, r_{0}\right]\right) \cup \mathcal{A}_{2}$. We observe that all of $\left.f_{\theta}\right|_{\Omega},\left.g_{\theta}\right|_{\Omega}$ and $\left.\left(f_{\theta}^{2}+g_{\theta}^{2}\right)\right|_{\Omega}$ are bounded maps. Therefore, $X_{\theta}\left(\Sigma_{\theta}\right)$ is a bounded subset of $\mathbb{R}^{2}$.

On the other hand, the relation $\operatorname{DX}_{\theta}(q)=R_{\theta} \cdot \operatorname{DX}\left(R_{-\theta}(q)\right) \cdot R_{-\theta}$, implies that

$$
\Gamma_{\theta}=R_{\theta}(\Gamma) \quad \text { where } \quad \Gamma=\left\{p \in \mathbb{R}^{2}: \operatorname{Trace}(\mathrm{DX})(p)=0\right\}
$$

Let us prove item (a) of Theorem D. Suppose that $X$ is a non-injective polynomial map such that $\operatorname{Spec}(X) \cap\{0\}=\varnothing$ and let $\Sigma=R_{-\theta}\left(\Sigma_{\theta}\right) \subset \Gamma$. Since $\Sigma_{\theta}$ is noncompact, $\Sigma$ is non-compact. On the other hand, $X(\Sigma)=R_{-\theta}\left(X_{\theta}\left(\Sigma_{\theta}\right)\right)$ is a bounded subset of $\mathbb{R}^{2}$ which implies that all $\left.f\right|_{\Gamma},\left.g\right|_{\Gamma}$ and $\left.\left(f^{2}+g^{2}\right)\right|_{\Gamma}$ are not proper maps (because $\Sigma \subset \Gamma$ ). Therefore if one of $\left.f\right|_{\Gamma}$ or $\left.g\right|_{\Gamma}$ is a proper map, $X$ is injective.

Let us prove item (b) of Theorem D. By Proposition 5 if $X$ is an injective polynomial map such that $\operatorname{Spec}(X) \cap\{0\}=\varnothing$, then $\left.\left(f^{2}+g^{2}\right)\right|_{\Gamma}$ is a proper map. On the other hand, if $X$ is a non-injective polynomial map such that $\operatorname{Spec}(X) \cap\{0\}=\varnothing$, proceeding as in item (a) we conclude that $\left.\left(f^{2}+g^{2}\right)\right|_{\Gamma}$ is a not proper map.

\section{Examples and Applications. Keller Maps}

Example 1 Let $X=\left(f_{x}, f_{y}\right): \mathbb{R}^{2} \rightarrow \mathbb{R}^{2}$ be the gradient of a polynomial map $f: \mathbb{R}^{2} \rightarrow \mathbb{R}$. If $X$ is an orientation preserving and locally diffeomorphic map, then Theorem $C$ implies that $X$ is injective.

Indeed, as the matrix $\operatorname{DX}(p)$ is non-singular and symmetric for all $p \in \mathbb{R}^{2}$, its eigenvalues are real and non-null. As $X$ preserves orientation, $\operatorname{Spec}(X) \subset(-\infty, 0)$ or $\operatorname{Spec}(X) \subset(0,+\infty)$. Therefore, by Theorem $\mathrm{C}, X$ is injective.

Example 2 Let $n>1$ be a natural number and let

$$
\begin{gathered}
f=x-2 y+y^{n} \\
g=x-y+y^{n}
\end{gathered}
$$

Then $X=(f, g): \mathbb{R}^{2} \rightarrow \mathbb{R}^{2}$ is an injective Keller map. Moreover, if $n$ is even, $\operatorname{Spec}(X)=\mathbb{S}^{1} \cup(\mathbb{R} \backslash\{0\})$. That is, $\operatorname{Spec}(X)$ is the biggest possible for Keller maps

Notice that Trace $(\mathrm{DX})(x, y)=n y^{n-1}$. This means that

$$
\Gamma=\{(x, y): \operatorname{Trace}(\mathrm{DX})(x, y)=0\}=\{(x, y): y=0\}
$$

We check that both $\left.f\right|_{\Gamma}$ and $\left.g\right|_{\Gamma}$ are proper maps, then by Theorem $\mathrm{D}, X$ is injective. The characteristic polynomial of $\operatorname{DX}(x, y)$ is $\lambda^{2}-n y^{n-1} \lambda+1$. Therefore $\operatorname{Spec}(X)$ is given by all the numbers $1 / 2 \cdot\left(n y^{n-1} \pm \sqrt{n^{2} y^{2 n-2}-4}\right), y \in \mathbb{R}$. It is easy to see that $\mathbb{R} \backslash\{0\} \subset \operatorname{Spec}(X)$ if $n$ is even and then $\operatorname{Spec}(X)=\mathbb{S}^{1} \cup(\mathbb{R} \backslash\{0\})$ (because $\operatorname{Spec}(X)$ 
is a connected set of $\left.\mathbb{R}^{2}\right)$. By taking an odd natural $n \geq 3$, in this example, we get that $\operatorname{Spec}(X)$ is made up of the union of the sets $\mathbb{S}^{1} \cap\{(x, y): x>0\}$ and $(0, \infty)$.

The following example (see [3]) shows the existence of analytic non-injective Keller maps.

Example 3 Let

$$
\begin{aligned}
& f=\sqrt{2} e^{x / 2} \cos \left(\frac{y}{e^{x}}\right) \\
& g=\sqrt{2} e^{x / 2} \sin \left(\frac{y}{e^{x}}\right) .
\end{aligned}
$$

Then $X=(f, g): \mathbb{R}^{2} \rightarrow \mathbb{R}^{2}$ is a non-injective analytic Keller map.

It is easy to check that $X$ is a Keller map. It is also non-injective, for instance, observe that for all $k \in \mathbb{Z}$ and all $y \in \mathbb{R}, X(0, y+2 k \pi)=\sqrt{2}(\cos (y), \sin (y))$.

Let us proceed to describe the adjacent half-Reeb components of $X_{f}$ and $X_{g}$. In fact, both $X_{f}$ and $X_{g}$ have infinitely many adjacent half-Reeb components (see Definition 4). In fact, observe that $f$ vanishes along all curves of the form

$$
C_{k}(t):=\left\{(x, y): x=t, y=(\pi / 2+\pi k) \cdot \mathrm{e}^{t}\right\}, \quad t \in \mathbb{R}, k \in \mathbb{Z} .
$$

Also $f(0, y)=\sqrt{2} \cos (y)$ and then $\frac{\partial}{\partial y} f(0, y)$ vanishes only once in the segment

$$
S_{k}:=\{(x, y): x=0, \pi / 2+\pi k \leq y \leq \pi / 2+\pi(k+1)\}
$$

that connects $C_{k}(0)$ and $C_{k+1}(0)$. We observe that $f$ is bounded in the semi-plane $\{(x, y): x \leq 0\}$ and unbounded in $\{(x, y): x>0\}$. In this way, for all $k \in \mathbb{Z}$, $X_{f}$ has a half-Reeb component $\mathcal{A}_{k}$ bounded its non-compact edges $\left\{C_{k}(t), t \leq 0\right\}$ and $\left\{C_{k+1}(t), t \leq 0\right\}$ and its compact edge $S_{k}$. All consecutive pairs $\left\{\mathcal{A}_{k}, \mathcal{A}_{k+1}\right\}$ are adjacent. Similarly, $X_{g}$ has a Half-Reeb component $\mathcal{B}_{k}$ between consecutive curves of the form

$$
D_{k}(t):=\left\{(x, y): x=t, y=(\pi+\pi k) \cdot \mathrm{e}^{t}\right\}, \quad t \in \mathbb{R}, k \in \mathbb{Z}
$$

and the segment

$$
T_{k}:=\{(x, y): x=0, \pi+\pi k \leq y \leq \pi / 2+\pi(k+1)\},
$$

and all consecutive pairs $\left\{\mathcal{B}_{k}, \mathcal{B}_{k+1}\right\}$ are adjacent. Observe that the curve $D_{k}(t), t \leq$ 0 is the only semi-trajectory of $X_{g}$ which is completely contained in the half-Reeb component $\mathcal{A}_{k}$ of $X_{f}$.

Example 4 If $X=(f, g): \mathbb{R}^{2} \rightarrow \mathbb{R}^{2}$ is the Pinchuck non-injective polynomial map (see [15]), then, by Theorem $C, \operatorname{Spec}(X)$ meets the real line at arbitrarily small positive and negative numbers.

Let us see this by directly describing the foliation induced by $X_{f}$, where

$$
f=(x y-1)(x(x y-1)+1)+(x(x y-1)+1)^{2}\left((x y-1)^{2}+y\right)
$$


After an extensive use of symbolic computation, one may see that:

$(4,1)$ for every $c<0,\{f=c\}$ is connected;

$(4,2)$ for every $c \geq 0,\{f=c\}$ has 3 connected components;

$(4,3)$ the set $\{f \leq 0\}$ is usually known as a Reeb component of $X_{f}$ and the set $\{f<0\}$ is homeomorphic to a disc;

$(4,4)$ for every $c \in \mathbb{R}$, a connected component of $\{f=c\}$ can approach to infinity, only at one of the following directions: the positive $x$-axis, the negative $x$-axis and the negative $y$-axis.

$(4,5)\{f=0\}$ consists of 3 connected components $A_{1}, A_{2}, A_{3}$ such that

- $A_{1}$ is contained in $\{(x, y): x y>0\} \cup\{(0,0\}$ and approaches infinity in the directions of the positive $x$-axis and the negative $x$-axis.

- $A_{2}$ is contained in $\{(x, y): x<0, y<0\}$ and approaches infinity in the directions of the negative $x$-axis and the negative $y$-axis.

- $A_{3}$ is contained in $\{(x, y): y>0\}$ meeting the $x$-axis exactly at $(1,0)$; it approaches infinity in the directions of the positive $x$-axis and the negative $y$-axis.

$(4,5)$ the Reeb component $\{f \leq 0\}$ contains 2 half-Reeb components, one of which approaches the positive $x$-axis and the other the negative $x$-axis. By observing, as in the proof of Theorem $\mathrm{C}$, the gradient vector field $\left(f_{x}, f_{y}\right)$ along this halfReeb components, it can be seen that there are sequences $\left\{a_{n}\right\}$ and $\left\{b_{n}\right\}$ such that, for all $n, f_{x}\left(a_{n}\right)>0, f_{y}\left(a_{n}\right)=0=f_{y}\left(b_{n}\right), f_{x}\left(b_{n}\right)<0$ and moreover $f_{x}\left(a_{n}\right) \rightarrow 0$ and $f_{x}\left(b_{n}\right) \rightarrow 0$. In other words, $\operatorname{Spec}(X)$ meets the real line at arbitrarily small positive and negative numbers.

Example 5 Let $X=(f, g): \mathbb{R}^{2} \rightarrow \mathbb{R}^{2}$ be an orientation preserving and locally diffeomorphic polynomial map. If $X$ is injective then it follows, from the main result of either of the papers [1] and [12], that $X$ is a diffeomorphism. Therefore, $X_{f}$ and $X_{g}$ have no Reeb components.

In fact, if $X_{f}$ has a hRc, say $\mathcal{A}$, then, we may see that $X(\mathcal{A})$ is bounded. This is not possible because $\mathcal{A}$ is unbounded and $X$ is a diffeomorphism.

Acknowledgements The first author was partially supported by FAPESP grant number 99/01442-8; the second author by a PRONEX/FINEP/MCT grant number 76.97.1080.00; and the third author by a DGES grant number PB96-1153 and a CIRIT grant number 2001SGR 00173.

\section{References}

[1] A. Bialynicki-Birula and M. Rosenlicht, Injective morphisms of real algebraic varieties. Proc. Amer. Math. Soc. 13(1962), 200-203.

[2] L. A. Campbell, Unipotent Jacobian matrices and univalent maps. Contemp. Math. 264(2000), 157-177.

[3] M. Chamberland and G. Meisters, A mountain pass to the Jacobian Conjecture. Canad. Math. Bull. 41(1998), 442-451.

[4] F. Dumortier, Singularities of vector fields on the plane. J. Differential Equations 23, 1(1977), 53-106.

[5] A. van den Essen, Polynomial automorphisms and the Jacobian conjecture. Progr. Math. 190, Birkhauser Verlag, Basel, 2000.

[6] R. Fessler, A proof of the two dimensional Markus-Yamabe Stability Conjecture and a generalization. Ann. Polon. Math. LXII(1995), 45-74. 
[7] M. Golubitsky and V. Guillemin, Stable mapings and their singularities. Grad. Texts in Math. 14, Springer-Verlag, 1973.

[8] E. A. González, Generic properties of polynomial vector fields at infinity. Trans. Amer. Math. Soc. 143(1969), 201-222.

[9] C. Gutierrez, Smoothability of Cherry flows on two-manifolds. Lecture Notes in Math. 1007(1981), 308-331.

[10] C. Gutierrez, A solution to the bidimensional global asymptotic stability conjecture. Ann. Inst. Henri Poincaré 12(1995), 627-671.

[11] W. Kaplan, Regular curve-families filling the plane II. Duke Math. J. 8(1941), 11-46.

[12] K. Kurdyka, Injective endomorphisms of real algebraic sets are surjective. Math. Ann. 313(1999), 69-82.

[13] L. Markus and H. Yamabe, Global stability criteria for differential systems. Osaka Math. J. 12(1960), 305-317.

[14] D. J. Newman, One-to-one polynomial maps. Proc. Amer. Math. Soc. 11(1960), 867-870.

[15] S. Pinchuck, A counterexample to the strong Jacobian conjecture. Math. Z. 217(1994), 1-4.

Departamento de Matemáticas

IBILCE-UNESP

São José do Rio Preto (SP)

Brazil

e-mail:milton@mat.ibilce.unesp.br

Departament de Matemàtiques

Universitat Autònoma de Barcelona

08193 Bellaterra

Barcelona

Spain

e-mail: jllibre@mat.uab.es
ICMC-USP

São Carlos \& IMPA

Rio de Janeiro

Brazil

e-mail: gutp@icmc.sc.usp.br 